\title{
Die Zahl 666, ein Hinweis auf Trajan.
}

\author{
Von Prof. Dr. W. Hadorn in Bern (Schweiz).
}

Man wird nicht behaupten können, daß die auch in dieser Zeitschrift reichlich benützte und wertvolle Aussprache der Forscher über den Sinn der rätselhaften Stelle Apc 13,17.18 und besonders über die Bedeutung der Zahl $666 \mathrm{zu}$ einem völlig befriedigenden und allgemein anerkannten Ergebnis geführt hätte, wenn schon die Deutung der Zahl auf Nero sich weitgehender Zustimmung erfreut. Aber diese Lösung leidet einmal an einem gewissen formalen :Mangel, daß sie nur auf Grund des hebräischen Alphabetes herausgebracht werden kann. Das kann man aber von den kleinasiatischen, nur griechisch redenden Empfängern der Apokalypse einfach nicht erwarten. Zum andern war diese Deutung, soweit wir urteilen können, den Alten nicht bekannt. Endlich sagt sie den Lesern nicht das, was sie eigentlich wissen sollten. Dieser letztere Mangel, der einem gewissen Versagen dieser beliebten Lösung gleichkommt, scheint mir noch größer zu sein, als der rein formale Fehler der Deutung auf Nero. Soll der Versuch einer Deutung dieser Stelle und einer Berechnung der Zahl 666 irgendwie mit Aussicht auf Erfolg unternommen werden, so muß man sich ganz klar darüber sein, was der Verfasser dem Leser sagen wollte.

Auf die schwierigen literarischen Probleme dieses Kapitels kann ich an dieser Stelle nicht eingehen, sie sind auch für die in Frage stehenden beiden Verse zunächst sekundär. Nur daran sei erinnert, was keinem Leser der Apokalypse entgeht, daß, wie das 13. Kapitel innerhalb des Ganzen einen Höhepunkt bedeutet, vorbereitet durch die Siegel- und Posaunenvisionen und die Erscheinung des Drachen auf Erden, so auch diese Schlußverse den Höhepunkt des 13. Kapitels darstellen: Die geheimnisvollen Schlußworte mit der Mahnung an die Leser, die Zahl des Tieres auszurechnen, machen die Absicht des Verfassers deutlich, ihnen eine wichtige Mitteilung zukommen zu lassen und ihnen einen Wink zu geben, der für sie in ihrer Lage von unmittelbarster Bedeutung ist. Denn zum ersten Male in dem Buche wird - vorausgesetzt, daß die Deutung der Stelle richtig ist,- der Name einer menschlichen Persönlichkeit genannt, zwar. immerhin in verhüllter Form, aber doch soweit bekanntgegeben; daß er auszurechnen ist und gefunden werden kann. 
An Winken und Hinweisungen auf zeitgeschichtliche Ereignisse hat es auch, abgesehen von den in diesem Zusammenhang außer Betracht fallenden Sendschreiben, in den vorangegangenen Visionen nicht gefehlt. Der Name Euphrat 9,14 mußte die Leser des Buches aufhorchen machen. Die Erwähnung der Stadt, in welcher "ihr Herr gekreuzigt ist", 11,8, war ein deutlicher Hinweis auf Jerusalem und auf das; was dort geschehen ist. Vielleicht könnte man noch andre zeitgeschichtliche Anspielungen namhaft machen. Allein bisher war noch nie so deutlich auf einen menschlichen Namen und auf eine menschliche Persönlichkeit gleichsam mit dem Finger und in einem zum Flüstern herabsinkenden Ton der Stimme gezeigt und von ihr gesprochen worden, wie am Schluß dieses 13. Kapitels. Wenn das Buch der Apokalypse für die Leser irgendwie eine für die Gegenwart bezügliche Bedeutung und Beziehung haben sollte, so war sie hier gegeben. Die Leser mußten die Empfindung haben: jetzt redet der Prophet so deutlich wie noch nie! Es gibt in der Offenbarung nur noch eine Stelle, die denselben unmittelbaren Gegenwartswert hat und beim Lesen dieselbe Empfindung auslöst. Es ist die Stelle 17,8.9, in welcher mit fast gleichlautenden Worten an die Weisheit der Leser appelliert wird.

Wir können das vielleicht deshalb weniger mit- und nachempfinden, weil für uns diese zeitgeschichtlichen Beziehungen nur noch ein geschichtliches Interesse haben. Für die Leser und Empfänger der Offenbarung verhielt es sich ganz anders. Wohl bleibt der Verfasser nicht bei der Gegenwart stehen. - Ihm war das; was in der nächsten Zukunft geschehen sollte, die.Parusie des Christus und das Kommen des Reiches Gottes, weit wichtiger. Aber gerade darum hatte für ihn diese Zahl 666 ein starkes Interesse, fand er doch in ihr den Schlüssel zum Verständnis seiner Zeit und der. Zeichen, Ereignisse und Geschehnisse seiner Zeit, gleichsam das Pfand und die Garantie, daß die Erfüllung der herrlichen Erwartungen und der seligen Hoffnungen nicht mehr weit sei. War der Antichrist schon da, dann konnte der Christus nicht mehr ferne sein. So merkwürdig es auch erscheinen mag, daß man aus der Ankunft des Antichrists auf die Nähe des Christus schloß, so menschlich verständlich ist dieser Schluß; vgl. 2 Thess $2,1-12$ ! Wie man bei jeder Steigerung des Fiebers im Verlauf einer akuten Krankheit auf die Nähe der Krisis schließt, weil es, wie man glaubt, einfach nicht mehr schlimmer werden kann, wie man in dieser Kriegszeit den Frieden so oft für nahe glaubte, als man eine weitere Steigerung und Anspannung der feindlichen Kräfte für ausgeschlossen hielt, so weckten auch in der Geschichte der Kirche und der Religion 
nicht die Zeiten einer friedlichen und stillen gedeihlichen Entwicklung des kirchlichen und religiösen Lebens die Hoffnung der Wiederkunft Christi, sondern vielmehr die Zeiten der Gärung, der Kämpfe, des Niedergangs und des Abfalls, die Zeiten, in denen sich so etwas wie ein Greuel an heiliger Stätte wiederholt. Das war ebenso die Auffassung Jesu (Mt 24) wie des Paulus 2 Thess $2,1-12$, wie es mit aller Deutlichkeit auch der I Johannesbrief 2,18 ausspricht.

Wir sind nun allerdings in den bisherigen Ausführungen von einer Voraussetzung ausgegangen, die erst noch bewiesen werden muß, sie wird zwar von einer großen Anzahl von Forschern geteilt, - daß sich unter der Zahl 666 eine geschichtliche Persönlichkeit verbirgt. In der Zahl muß daher der Schlüssel zum Verständnis dieses Kapitels gesucht werden.

Auf die Frage nach der Integrität des Kapitels brauchen wir nicht einzugehen. Die Überleitung von Vers $17^{\mathrm{a}}$ zu unserer Stelle ist gut und vẹrständlich. Die Angabe, daß niemand kaufen und verkaufen könne,

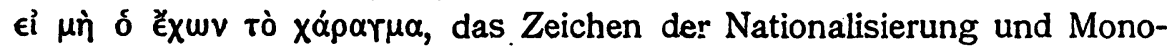
polisierung des Handels in der antichristlichen Notzeit, gibt dem Verfasser die Gelegenheit, seinen Lesern einen geheimen Wink bezüglich der zu erwartenden Persönlichkeit des Antichrists zu geben.

Auch die Schwankungen des Textes haben auf die Lösung keinen wesentlichen Einfluß. Die von Nestle adoptierte lesung von Vers 17

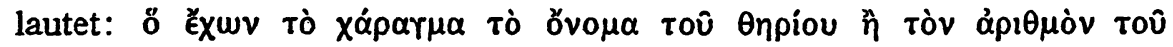
ठvó

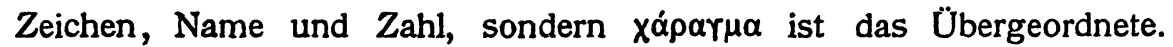
Man muB das Zeichen haben, welches entweder im Namen oder in

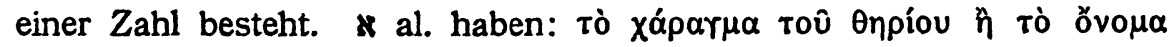
aủrov̂. Hier wird in der 2. Hälfte die Zahl weggelassen und in der ersten Hälfte Zeichen und Name einander gegenübergestellt! $X \alpha_{\alpha}-$ par $\mu \alpha$ gibt nun zunächst nur die Technik der Ausführung an, daß es eingeritzt, eingeschnitten oder aufgeprägt sei, also ein aufgedrucktes oder

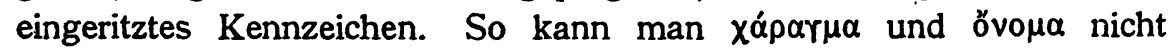
wohl nach dieser Variante einander gegenüberstellen, denn der Name

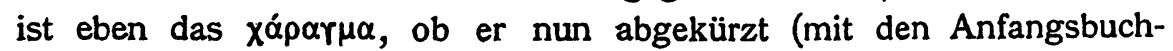
staben), oder voll ausgeschrieben oder symbolisch durch ein Geheimzeichen wiedergegeben wird. Holtzmann u. a. haben an die römischen Münzen erinnert, die ein Bild, z. B. den Kopf des Herrschers, und eine Inschrift aufweisen. Nun kann man sich schon vorstellen, daß auch ein Kopfbild wie eine Figur eingeritzt und eintätowiert werden kann, wie man das noch heute bei Soldaten und Matrosen aller Völker beob- 
achtet. Dieses Verfahren der Tätowierung eines Kopfbildes oder einer Figur ist aber ziemlich umständlich und für eine Massenanwendung nicht geeignet. Dagegen ist der Aufdruck des Namenszuges, besonders in abgekürzter Form, oder eines Tierzeichens leichter durchführbar. Ich erinnere daran, wie noch heute in der Türkei der Namenszug des Sultans. zugleich das Wappenzeichen auf den Fahnen, Siegeln, Dokumenten und Briefmarken ist, oder wie in monarchischen Ländern einzelne Regimenter den Namen eines Herrschers vollständig oder als Monogramm auf den Kopf bedeckungen, Achselklappen oder am Arm tragen. Die rezipierte Lesung ist trotz der scheinbaren Überladung

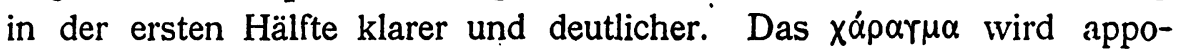
sitionell bestimmt. Die Anhänger des Tieres haben ein Malzeichen, nämlich den Namenszug des Tieres, also des Antichrists, sei es ausgeschrieben, sei es als Monogramm.

Schwieriger ist die 2. Vershälfte. Sie kann beigefügt sein zur Überleitung auf Vers 18. Das wäre dann wahrscheinlich, wenn Vers 18 eine Glosse ist. Es ist aber ebensogut denkbar, daß auch die Zahl des Namens, statt des Monogramms als abgekürzte Schreibung dienen sollte. So geben einige Varianten zu Vers 18 statt der ausgeschriebenen Zahl die 3 Buchstabenzeichen $\chi \xi \xi$. So gut wie man sich aus religiösen superstitiösen Vorstellungen den Namen oder das Zeichen eines Gottes aufdrữcken ließ, konnte man sich auch die Zahl des Namens, d. h. die Summe der Zahlenwerte seiner Buchstaben aufdrücken lassen. Ob sich nun dieser Brauch nachweisen läßt oder nicht, so ist doch auch in der 2. Hälfte des mit $\epsilon$ ì eingeleiteten Nebensatzes die Meinung des Verfassers klar und deutlich. Er sagt: die Anbeter des Tieres haben ein Malzeichen, entweder seinen Namen, oder die Zahl seines Namens: Dafür spricht 15,2. Die Überwinder haben Widerstand geleistet dem Tier, seinem Bilde und der Zahl seines Namens, d. h. sie haben sich geweigert, das Bild anzubeten und sich die Zahl seines Namens aufdrücken zu lassen. Nach dieser Stelle kann es sich nicht um die in Worten ausgeschriebene Zahl, sondern nur um die 3 Buchstaben $x \xi s$ handeln.

Nun folgt der berühmte 18. Vers, der die Leser auffordert, diese

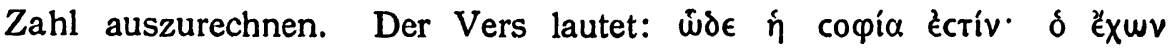

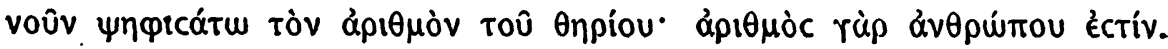

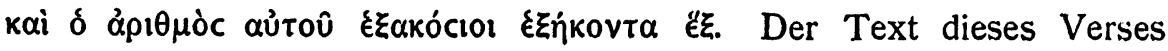
ist in der Überlieferung ziemlich konstant, mit Ausnahme von zwei allein in Betracht kommenden Varianten $x \xi_{5}$ statt der ausgeschriebenen

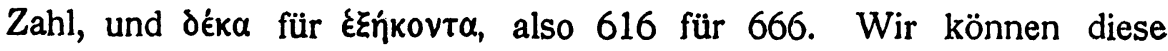


Varianten vorläufig auf sich beruhen lassen. Vorderhand ist der Sinn

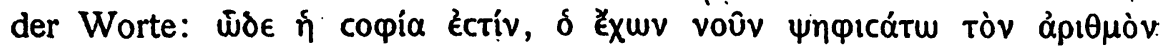

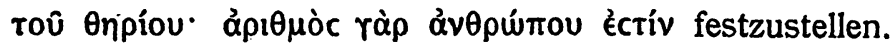

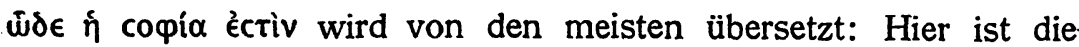
Weisheit vonnöten, nämlich die Weisheit; welche allein imstande ist, in das Geheimnis dieser apokalyptischen Weissagung einzudringen, welche der gewöhnliche Sterbliche und der außerhalb des christlichen Kreises Lebende nicht hat. Ich sehe auch keine Nötigung, von dieser natürlichen Deutung abzugehen und das copí in gnostischem Sinne $\mathrm{zu}$ erklären. Der Apokalyptiker will aber auch kaum sagen, daß es zur Ausrechnung dieser Zahl lediglich einer gewissen geistigen Begabung bedürfe, so daß jeder einigermaßen begabte und gebildete Mensch - dieses Zahlenrätsel lösen könne. Das wäre das gerade Gegenteil von dem, was er sagen will. Um ein bloßes Zahlenrätsel durch Gematria $\mathrm{zu}$ lösen, braucht es nur 'eine gewisse Findigkeit und Geduld, nicht aber cọi $\alpha$. Er appelliert vielmehr an die tiefere Einsicht der Christen, die erleuchtete Augen haben müssen, um. das Rechte $z u$ finden. Denn wenn, vorausgesetzt, daß das Ausrechnen auf die Gematria geht, der Name so gefunden werden muß, daß eine Gleichung mit mehreren Unbekannten entsteht: $666=\mathrm{x}+\mathrm{y}+\mathrm{z}+\ldots$. ., so mußte er sich selbst sagen, daß auf diesem Wege mehrere Namen gefunden werden konnten. So bedarf es immerhin einer göttlichen Weisheit, um den rechten zu finden. Der Satz ist durchaus dem andern parallel, 17,9:

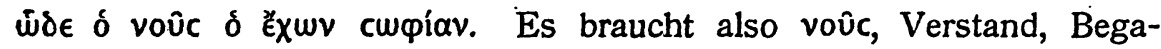
bung, aber auch einer Leitung des voûc durch die copía:

Wem nun diese Weisheit zuteil geworden ist, der, so fordert der Apokalyptiker weiter auf, rechne die Zahl des Tieres aus. $\Psi_{\eta \varphi i ́}$ íı

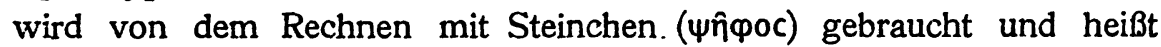
ausrechnen. Auch in der Wahrsagerkunst brauchte man solche Steinchen zum Berechnen künttiger oder verborgener Dinge. Man brauchte es auch als Ausdruck für die Stimmabgabe bei Wahlen und Prozessen, indem.man das verdammende oder freisprechende Urteil auf ein Steinchen schrieb. So kam Voelter auf die seltsame Deutung: der Leser

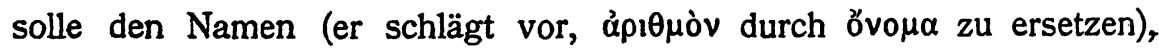
um den es sich handelt, auf ein Steinchen schreiben, und dieses in die Urne werfen und damit sein Urteil darüber abgeben, welche Person gemeint sei, ein umständliches Verfahren, welches zum Glücke der Verfasser seinen Lesern nicht zugemutet hat. Der Sinn der Stelle scheint mir doch klar zu sein: Wer Verstand hat, soll die Zahl des Tieres ausrechen, so daß er den Namen herausbringt, wenn anders. 
in Vers 17 der Name und die Zahl äquivalent sind und sich decken und die Zahl einen Namen bedeutet. Ein anderes Ausrechnen der Zahl gibt es nicht, wenn die Zahl gegeben ist. Wäre die Zahl die

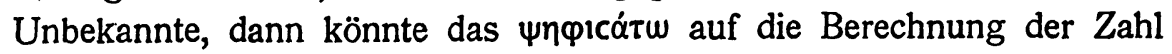
aus bestimmten andern Faktoren gehen. $\mathrm{Da}$ sie aber gegeben ist, kann es sich nur um eine Berechnung der Summanden handeln.

Eines wird an dieser Stelle nicht gesagt, wier der Verfasser zu dieser Zahl gekommen ist. Diese Frage darf nicht umgangen werden. Aber sie kann vorderhand noch zurückgestellt werden, da sie für die Leser gleichgültig war. Dagegen sollten wir wissen, welche Art der Berechnung vorausgesetzt wird? Möglich ist

1. die Zerlegung der Zahl als einer Summe von einzelnen Summanden (Gematria),

2. Berechnung der Zahl durch Zurückführen auf eine Basiszahl,

3. Deutung der Zahl nach symbolischen Merkmalen.

Die meisten Erklärer entscheiden sich für die Gematria. Die Umrechnung von Namen durch Addition der Zahlenwerte der einzelnen Buchstaben in eine Zahl und umgekehrt die. Ausrechnung eines Namens aus einer Zahl durch Zerlegung in die Summanden war damals ein beliebtes Verfahren. Nur sollte man wissen, welches Alphabet zugrunde gelegt werden muß, das hebräische oder das griechische. Das lateinische kommt nicht in Betracht.

Bevor aber dieses Verfahren mit Aussicht auf Erfolg angewendet werden kann, sollte man ferner wissen, was eigentlich gefunden werden soll. Denn aus einer Zahl kann man alles Mögliche und Unmögliche herausrechnen. Nun gibt der Verfasser noch einen Wink, der. gleichsam die Probe aufs Exempel für die Richtigkeit der Berechnung in

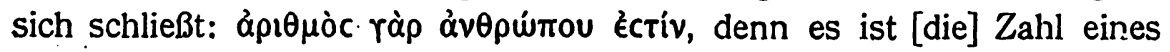
Menschen, was wiederum auf verschiedene Weise übersetzt und erklärt werden kann; entweder so, daß die Zahl den Namen eines Menschen bedeutet, sei es eines jetzt lebenden oder eines zukünftigen, oder, wie Verschiedene vorschlagen, als eine Zahl, wie sie Menschen gebrauchen. So u. a. C. Clemen (ZNW II 112.)

Die Vertreter dieser Übersetzung berufen sich auf die Stelle 21,17,

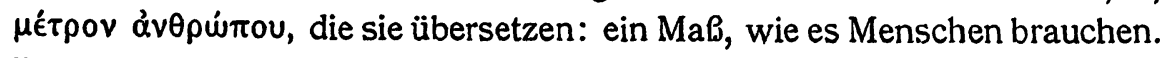
Das ist aber nicht ganz richtig, indem an dieser Stelle noch beigefügt ist: ö écțı àrré̉ov, was sagen würde: oder genauer eines Engels. Nun soll nicht betont werden, daß Menschen resp. Engel dieses Maß brauchen, sondern daß die Länge der Elle von einem Menschen genommen sei, vom Ellbogen bis zur Spitze des mittleren Fingers, eine 
Bezeichnung, die angesichts der verschiedenen Längenmaße, die mit

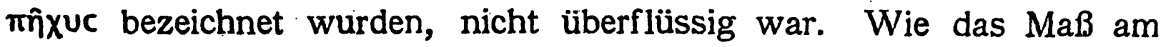
Menschen gemessen und abgenommen wird, so kann auch die Zahl des Menschennamens an einem Menschen abgenommen werden, in diesem Falle an einem bestimmten Menschen. Denn der Ausdruck: Zahl, wie sie Menschen brauchen, ist sinnlos. Es gibt gar keine anderen Zahlen! Oder was wären dann die „ungebräuchlichen“'Zahlen? Allein zu dieser Übersetzung muß man eben seine Zuflucht nehmen, wenn man hinter 666 nicht einen Menschen, sondern ein Kollektivum, eine Sache, vermutet. In diesem Falle; wenn z. B. 666 das Reich oder so etwas bedeutet, kann man mit der Zahl eines Menschen nichts anfangen. So scheiden sich die Deutungen, die vorgeschlagen worden sind, in zwei Gruppen, Deutungen auf den Namen eines Menschen und Deutungen auf ein Kollektivum. Diese beiden Deutungen entsprechen auch den beiden einander ausschließenden Deutungen des ersten Tieres in Kap. 13 auf eine einzelne geschichtliche Persönlichkeit (das tödlich verwundete Haupt) oder auf das Reich'als Kollektivum ohne nähere Bezugnahme auf einen einzelnen Fürsten. Ich halte dafür, daß die bisherigen Untersuchungen über die beiden Tiere und über die Worte

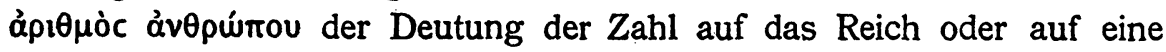
Kollektivgröße nicht günstig sind. Die Hervorhebung des Kaiserkultus durch Betonung der Anbetung des Bildes nötigt, unter dem Onpíov eine Persönlichkeit zu denken. Die Entscheidung liegt jedoch in der Deutung der Zahl 666, auf welche die Anlage des ganzen Kapitels zustrebt. Aber erst wenn sich eine einwandfreie Deutung der Zahl ergibt, die mit dem Vorhergehenden übereinstimmt, kann das Ergebnis als gesichert gelten. Weil bisher alle Deutungen auf einen Menschen nicht einwandfrei waren, deshalb wandten sich viele Forscher auch der andern Möglichkeit zu, die Zahl auf ein Kollektivum zu deuten.

Ich stelle nochmals die Frage: was soll der Leser eigentlich erfahren? $\mathrm{DaB}$ sich unter dem Tier etwas anderes verbirgt, das merkte auch der einfältigste Leser. Wahrscheinlich merkte er aber auch, daß das Tier in irgendeiner Form mit dem römischen Reich zusammenhängt. Die Deutung des „letzten Tieres“, oder des „Tieres“ schlechthin, und des kleinen Hornes bei Daniel auf das römische Imperium war für die palästinensischen Theologen ein Axiom (Schlatter, AT i. d. Joh-Apok. S. 89). Von dieser Auffassung kann die christliche Gemeinde kaum ganz unberührt geblieben sein. Aus dem Grunde war dem Leser damit keine besondere Offenbarung gegeben, wenn sich hinter der Zahl 666 nur der Name des Reiches versteckte. Was

Zeitschr. f. d. neutest. Wiss. 19. Jahrg. 1919/20.' 
mußte er wissen? Nach Vers 3-8 doch gewiß nur das eine, wer der Antichrist sein wird oder ist. Den Namen des Antichrists zu erfahren, darauf waren sie gespannt, und in dieser Erwartung haben sie das Kapitel gelesen. $\mathrm{DaB}$ das erste Tier der Antichrist ist, das haben wohl die meisten erraten. Wer aber der gedachte und erwartete Antichrist ist, das mußte ausgesprochen werden. Ob die vorgeschlagenen verschiedenen Deutungen dieser Fragestellung gerecht werden, muß im folgenden untersucht werden.

Nun noch die zurückgestelltè Frage: Welches ist der Ursprung der Zahl 666? Es sind auch hier zwei Möglichkeiten denkbar:

1. Entweder ist die Zahl erst durch Gematria entstanden, indem vom Verfasser der ins Auge gefaßte Name der betreffenden Persönlichkeit nach seinen Zahlenwerten ausgerechnet und eingesetzt worden ist, in der Meinung, daß der Leser die Summe dann auch wieder auf diesem Wege auflöse. Oder

2. die Zahl ist überliefert, wie die Zahl der $3^{1 / 2}$ Zeiten oder der 42 Monate, irgendwie und irgendwann entstanden, vom Apokalyptiker übernommen, in der Meinung allerdings, daß sie durch Gematria ausgerechnet, $d$. h. entziffert werden könnte. In diesem Falle wäre eine doppelte Berechnung der Zahl möglich, einmal nach ihrer ursprünglichen traditonellen und endgeschichtlichen Bedeutung, und sodann - nach der Absicht des Verfassers - nach ihrer zeitgeschichtlichen Verwertung. Er würde sie nicht aufgenommen haben, wenn er nicht die Überzeugung hätte, daß der Leser eben jetzt die Antwort auf die Frage nach der Person des antichristlichen Herrschers aus der Zahl entziffern könne.

Diese zweite Möglichkeit, daß eine alte traditionelle Zahl vorliege, muß aber abgelehnt werden. Wir haben nirgends irgendeine Spur von einer solchen Zahl. Und wenn man auch versucht, die Zahl 666 aus symbolischen Reflexionen abzuleiten, und die 3 Sechser als' ein typisches Zeichen des Antichrists aufzufassen, als Antitypus für die Zahl des Messias 888, welche die Sybille I 326-330 bringt [Incouc $=888$; $I=10, \eta=8, c=200, o=70, v=400, c=200$, total 888$]$, so ist erst recht die Frage erlaubt, ob nicht die Zahl 888 auf Grund und nach dem Vorgang der Zahl 666 gebildet ist. Ebenso verhält es sich mit dem Zahlzeichen $\chi^{\xi} \varsigma$, welches als Antitypos für die Abkürzung für Christus hingestellt worden ist. Zwischen dem ersten Buchstaben des Namens $X$ und dem letzten $\Sigma$ soll sich das $\xi$ als das schlangenähnliche Symbol des Drachen gedrängt haben, zum Zeichen daß $\chi \xi \varsigma$ das Zeichen des Antichrists sei. (Vgl. Spitta, Off. S. 388.) Allein auf diese recht künstliche Weise kann die Zahl nicht entstanden sein. 
Dazu kommt noch eins. Wäre die. Zahl alt gewesen, so hätte sie den Lesern der Apokalypse nichts genützt. Ihre Mitteilung hatte doch nur dann einen Sinn, wenn sie ihnen die Person des Antichrists enthüllte. Und warum greift der Verfasser überhaupt $\mathrm{zu}$ diesem Mittel? Doch gewiß nur, weil es höchst gefährlich war, den Namen zu nennen. Nur im Geheimen und nur gleichsam vermittelst einer durch Dechiffrierung zu entziffernden Geheimschrift durfte dieser Name angedeutet werden (vgl. Schlatter, AT i. d. Ap. S. 75).

Aus diesem Grunde bringt auch der Versuch von G. A. van den Bergh (ZNW 1912, S. 293) keine Lösung. Er schlägt in geistreicher Weise vor, die Zahl 666 als sogenannte Dreieckszahl abzuleiten, entstanden als Summe einer Reihe von $1+2+3+\ldots+36=666$,

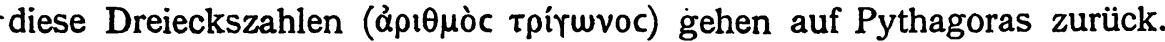
Aber auch 36 ist Dreieckszahl, entstanden aus der Reihe $1+2+3$ $+\ldots 8=36$. So könne man sagen, 666 sei in 36 und 36 in 8 enthalten, so daß als Grundzahl des Tieres die 'Orðoóc herauskomme, welche den Satz in 17;11 erklären würde: er ist der achte zu den sieben. Freilich führt er die Entstehung dieser Zahl auf alte gnostische Spekulationen zurück, ohne zwingende Gründe dafür vorbringen zu können, und ohne daß dem Leser damit etwas gesagt wird.

So bleibt nichts anderes übrig, als den Weg einzuschlagen, den der Verfasser vorschlägt, die Zahl auszurechnen und dabei den Wink $\mathrm{zu}$ beachten, $\mathrm{da} \beta$ es eines Menschen $Z$ ahl ist. Vielleicht daß gerade auf diesem Wege auch auf die Entstehung der Zahl ein Lichtstrahl fällt, der das über ihr schwebende Dunkel aufhellt. Ist nämlich nirgends eine Spur nachweisbar, daß es sich um eine alte, traditionell gegebene Zahl handelt, so muß die Zahl auf dem Wege entstanden sein, auf dem sie dann auch wieder eruiert werden kann. Ist die Beobachtung Schlatters (AT i. d. Apok. S. 74) richtig, daß bei den palästinensischen Theologen der Zahlenwert der biblischen Worte und Namen als ein Mittel galt, durch das der heil. Geist .Geheimnisse erkennbar macht, so lag es für einen Mann, der wie der Apokalyptiker seine Heimat in Palästina hat, nahe genug, sich dieses Mittels zu bedienen.

Die Lösungen, die für die Eruierung der Zahl vorgeschlagen worden sind, sind nun zahlreich genug, meistens aber ganz wertlos. Es hat keinen Sinn, sich mit allen $z \mathfrak{u}$ beschäftigen. Weitaus am meisten Anklang hat die Deutung auf Nero gefunden, gelingt es doch, aus den Zahlwerten des hebräischen Alphabetes den Namen des Kaisers Nero auszurechnen. Es entspricht auch diese Lösung dem Wink des Verfassers, daß die Zahl eines Menschen Zahl ist. 
Gegen diese Deutung hat man eingewandt, daß רop defektiv geschrieben sei, statt des üblichen-קיסר. Doch hat man einige Beispiele aus der Literatur beigebracht, nach welchen sich die defektive Schreibung auch nachweisen läßt (Vgl. Ewald, Apok. S. 263, Not. 1 und Buxt. Lex. Rabb.) Die Weglassung des Jod wäre dann vielleicht motiviert durch den Wunsch, eine symmetrische Zahl zu erhalten (Spitta 388).

Bedenklicher ist aber die Tatsache, daß dieser Berechnung hebräische Buchstaben zu Grunde gelegt werden müssen (S.11). Man macht dafür freilich geltend, die Gematria, die aus Babylonien stamme, sei bei den Juden sehr beliebt gewesen, und deshalb in hebräischen Buchstaben die natürliche Form, $n$ in dieser Form auch allein zulässig bei einem Buche, das hebräisch gedacht, nur gleichsam in griechischen Buchstaben geschrieben sei und die Kenntnis des Hebräischen voraussetze ${ }^{\text {" }}$ (so Holtzmann). Allein gerade die angerufenen Stellen 9,11 und 16,16 beweisen etwas andres. Der Verfasser findet es 9,11 für nötig,

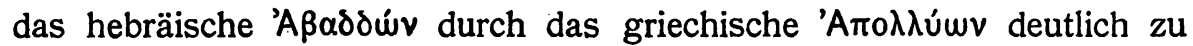
machen, und 16, 16 muß er ihnen geradezu sagen, daß Harmaggedon

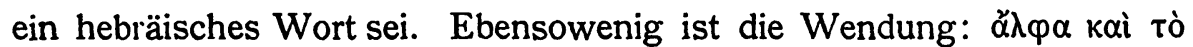
$\omega$ in 1,$8 ; 21,6 ; 22,13$ hebräisch gedacht. Daß, wie Zahn und Spitta betonen, die Gematria ursprünglich keine hebräische Kunst sei, fällt nicht wesentlich ins Gewicht. Ausschlaggebend ist meines Erachtens, daß den Lesern etwas zugemutet wird, was sie nicht leisten können. Spitta hat recht, wenn er die Behauptung Holtzmanns, für die Enträtselung der Zahl 666 sei die Anwendung von hebräischen Buchstaben allein zulässig, ablehnt. Die oben erwähnte Deutung des Namens Jesu durch die Sibylle 'Incouc $=888$ beruht auch auf griechischen Buchstaben.

$\mathrm{Zu}$ diesen schwerwiegenden Bedenken, welche bis jetzt noch von keinem Vertreter der Nerotradition entkräftet worden sind, kommt nun aber noch ein sachlicher Grund. Wir haben die Frage aufgeworfen: was mußten die Leser erfahren? Bedeutete die Zahl 666 wirklich den Kaiser Nero (Name und Titel!), so erfuhren sie also, daß der Antichrist der Kaiser Nero sei. Aber: war oder sein wird? Das erstere nützte ihnen nichts. Nero gehörte der Vergangenheit an. Also sein wird? Das heißt mit andern Worten: Nero werde als Antichrist wiederkommen, er werde, wenn er wiederkommen werde, der Antichrist sein. Diese Erwartung kombinierte man, wie wir glauben mit Recht, mit dem tödlich verwundeten Haupt, das wieder heil geworden ist. Aber, vorausgesetzt, man habe um jene Zeit die Nerosage in der dritten Phase, wie Bousset sagt, gekannt, dann brauchte man ja diesen Namen Nero gar nicht mehr so geheimnisvoll mitzuteilen. War sie aber nur dem 
Verfasser bekannt, so war die Mitteilung, daß der wiederkommende Nero der Antichrist sein werde, wiederum für die Leser des Buches nur eine halbe Offenbarung, etwa gleichwertig, wie wenn ein Schwärmer in dieser Zeit des Weltkrieges seinen Anhängern mitteilen würde, der Antichrist werde der wiederkommende Napoleon sein. Was helfen solche Weissagungen, wenn man daraus nichts Genaues und Bestimmtes erfahren kann, weder was den Zeitpunkt, noch was die geschichtliche Persönlichkeit betrifft! Um das zu sagen, hätte sich der Verfasser seine Berechnung und alle Geheimtuerei ersparen können. $\mathrm{Da}$ hat die Sibylle viel deutlicher von dem Muttermörder gesprochen.

Sollte die Deutung auf einen Namen einen Wert haben, dann mußte der Name in griechischen Buchstaben zu finden sein und zweitens eine bestimmte Persönlichkeit bezeichnen, welche die Leser kennen. Eine solche bestimmte. Persönlichkeit ist der wiederkommende Nero aber nur in dem Falle, wenn er in der Hülle einer andern Persönlichkeit auftritt, etwa so. wie Jesus von Johannes dem Täufer sagte, er sei der Elias. So, aber nur so, könnte man von jemand sagen, er sei der Nero. Dann aber mußten die Leser wissen, wer dieser Nero-Antichrist sei. (vgl. Voelter, Probl. 214).

Dazu kommt ein Drittes. Wir haben Grund zu der Annahme, die Apokalypse sei gegen Ende des Jahrhunderts geschrieben worden. Um diese Zeit waren aber bereits verschiedene falsche Nero aufgetreten, so daB die Annahme, der Antichrist werde der Nero sein, bei den Lesern höchstens Verwirrung anrichten konnte. Eine Hilfe oder eine Einsicht war ihnen dieser Name nicht. Und. welchen Sinn hatte es, so geheimnisvoll von dem wiederkommenden Nero zu reden. Einen Sinn hat diese Aufforderung des Verfassers nur, wenn der Leser wußte, wer dieser wiederkommende Nero ist.

Angesichts des Versagens der Deutungen auf eine geschichtliche Persönlichkeit nahm man die Zuflucht zu anonymen Deutungen! Es ist bezeichnend, daß dieselben sehr frühe auftreten. Kein Geringerer als Irenäus hat diesen Weg betreten. Er hat die für die Methode der Berechnung wichtige Bemerkung gemacht, der Name müsse aus dem griechischen Alphabet gefunden werden, es könnten aber auf diese Weise viele Namen herausgebracht werden. Er selbst schlägt 3 Namen

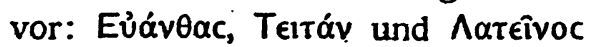

$$
\begin{aligned}
& E \quad v \quad \alpha \quad v \quad \theta \quad \alpha \quad c \\
& 5+400+1+50+9+1+200=666 \\
& T \quad \epsilon \quad \imath \quad \tau \quad \alpha \quad v \quad \text { (als Anspielung auf Titus) } \\
& 300+5+10+300+1+50=666
\end{aligned}
$$




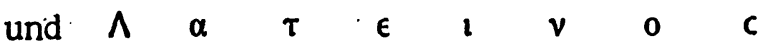 $30+1+300+5+10+50+70+200=666$}

Der Dreiervorschlag des Irenäus ist sehr interessant. Er beweist, daß man zur Zeit des Irenäus keine gesicherte Tradition mehr hatte. Hätten die Zeitgenossen des Johannes zur Zeit der Abfassung der Apokalypse die Meinung des Verfassers erraten und wäre es wirklich seine Meinung gewesen; daß der Name des wiederkehrenden Nero in dieser Zahl enthalten sei, dann hätte diese Deutung unmöglich so schnell vergessen werden können. Man sieht gar nicht ein, weshalb man diese Erwartung, die durch die Zahl 666 hätte befestigt werden müssen, nicht für alle Zukunft gerade mit dieser Zahl verband, besonders wenn es sich um Nero handelte! Man kann einwenden, daß die Berechnung mit hebräischen Buchstaben schuld an diesem Vergessen gewesen sei. Es ist aber noch ein andrer Grund denkbar, nämlich daß sich die ursprüngliche Annahme des Verfassers irrig erwies, indem der von ihm als Antichrist bezeichnete Herrscher sich in der Folgezeit gar nicht als der Antichrist herausstellte. So ging die ursprüngliche Deutung verloren. Die beiden Berechnungen des Irenäus: Euanthas und Teitan werden aber auch -von ihm selbst nicht ernst genommen, während die dritte

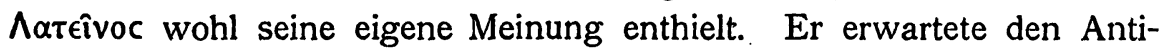
christ in der Person eines römischen Kaisers der Zukunft „quoniam novissimum regnum hoc habet vocabulum. Latini enim sunt qui nunc regnant, sed non in hoc nos gloriamur." Ganz sicher ist er aber wohl kaum, da er nach Adv. Haer. V 25, 2; 26, 1; annimmt, das Tier, d. h. der Antichrist werde dem bestehenden Reich der Latiner ein Ende bereiten. Ein Widerspruch ist das freilich noch nicht, da ja nach Apc 17 das Tier gegen Babylon zieht.

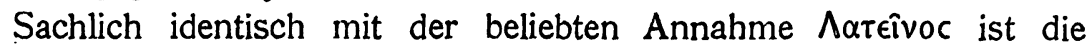
Deutung der Zahl auf קיסר רִּוֹמים (Vorschlag Weylandt), wo die Zahlenwerte auch $=666$ ergeben.

Da die Deutungen der Zahl 666 sowohl auf eine einzelne Persönlichkeit als auch auf ein Kollektivum nicht befriedigen, hat sich Spitta der Variante 616 zugewandt und die schon von Zahn als „ohne alle

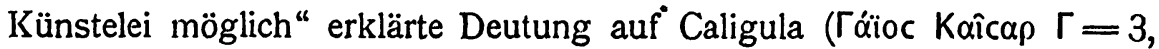
$\alpha=1, \imath=10,0=70, c=200, K=20, \alpha=1, \imath=10, c=200, \alpha=1$, $\rho=100$, total 616 ) befürwortet. Diese Lösung hat das für sich, daß sie das griechische Alphabet voraussetzt und daß sie auf eine bestimmte geschichtliche Persönlichkeit hinweist. Aber auch diese Lösung befriedigt nicht ganz. Einmal ist die Variante sekundär, und die Erklärung Spittas, der Redaktor habe die ursprüngliche Zahl 616 in 666 verändert, 
um den Namen Neros herauszubekommen, wenig einleuchtend. Vor allem aber, und das ist ausschlaggebend, entsteht eine komische Situation, wenn, wie Zahn richtig hervorhebt, der jüdische Apokalyptiker, der nach Spitta die Urschrift von c. 13 verfaßt haben soll, das griechische Alphabet benutzt, der christliche Redaktor aber, um die Zahl der inzwischen aufgetauchten Nerolegende anzupassen, das hebräische Alphabet herbeizieht, und das für griechische Leser.

Aber auch abgesehen von der bessern Bezeugung der Zahl 666 gegenüber $616 \mathrm{muß}$ ich die Zahl 666 für die ursprüngliche halten. Unzweifelhaft hat das symbolische Moment doch bei dieser Zahl eine Rolle gespielt,-obschon das Symbolische bei unserer Schreibung mit den drei Sechsern viel mehr in die Augen springt als bei der griechischen Schreibung: $600,60,6$, die nicht ins Auge fällt. Immerhin konnte sie doch einen Eindruck machen und $\mathrm{zu}$ phantastischen und spekulativen Erwägungen Anlaß geben, insofern auch in der griechischen Schreibung die Sechszahl eine Rolle spielt. Ich kann E. Vischer verstehen, wenn er (ZNW.IV 167-174). schon in der Zahhl selbst die Mitteilung eines Geheimnisses erblickt. Diese Zahl mit dem dreimaligen Vorkommen der Sechs muß in irgendeiner Weise das Wesen des Tieres ausdrücken. Auffallenderweise ist nun die Summe der Zahlenwerte des griechischen Wortes $\theta$ npíov ins Hebräische umgeschrieben genau 666. $n=400,7=200,\urcorner=10, i=6, j=50$, die Zahl des Tieres. Wenn man bedenkt, wie oft griechische Wörter als termini technici ins Hebräische hinübergenommen wurden, so kann man sich die durch die drei Sechser frappante Zahl wohl auf diesem Wege entstanden denken. Der Einwand, daß sie auf Grund des hebräischen Alphabetes entstanden sei, ist hier nicht stichhaltig, denn der Verfasser mutet ja seinen Lesern nicht $\mathrm{zu}$, das Wort Tier durch Rückschluß und Berechnung auf Grund des hebräischen Alphabetes zu finden, indem er ihnen selbst die Zahl 666 als Zahl des Tieres nennt. Auf welchem Wege er sie als Jude gefunden hatte, konnte den Lesern gleichgültig sein. Wenn nun Vischer meint, die Mitteilung dieser geheimnisvollen Zahl 666 sei eben die Hauptsache, so ist das nicht richtig. Denn mit der Zahl allein konnten die Leser nichts anfangen. Vischer will übrigens nicht leugnen, daß nebenbei die Aufforderung, die Zahl durch Gematrie auf eine historische Persönlichkeit $\mathbf{z u}$ berechnen, mit der Mitteilung verbunden gewesen sein könne. Das halte ich allerdings für tatsächlich richtig. Auf diese Weise kann die Zahl des Tieres von dem Verfasser - oder einem Zeitgenossen - durch Berechnung der Zahlwerte des hebräisch geschriebenen $\theta$ npiov = תריון gefunden worden sein, deren symbolische Bedeu- 
tung sich durch die drei Sechs alsbald aufdrängte. Dann aber drängte sich das Zweite auf, diese Zahl auf eine historische Persönlichkeit zu berechnen. Es würde hier eine Kombination von Isopsephie und Gematrie [Corssen ZNW III 238ff.] vorliegen, für die die Gleichung vorläufig lauten würde

Tier (ת), Onpiov) $=666=x$.

Diese $\mathrm{x}$ handelt es sich $\mathrm{zu}$ finden, und zwar in griechischen Buchstaben! Mehrere Forscher hahen sich abgemüht, den Namen Trajans in dieser Zahl wiederzufinden. Aberle schon 1872 in der Theol. Quartalschr. S. 144, ebenso Wabnitz (bei Voelter, Entstehung der Apok. S. 76). Sie waren aus sachlichen Gründen zu der Überzeugung gelangt, daß die Apokalypse auf Trajan hinweisen müsse. Aber ihre Berechnungen schlugen fehl. Sie schrieben תריון, oder mit Weglassung des s תרינון. Allein das $T$ muß mit 0 und nicht mit $n$ wiedergegeben werden. Voelter addierte die Worte Trajanus Hadrianus, des vollen Namens für Hadrian, und kam auf die beiden Summen $331+385=666$. Und doch waren Aberle und Wabnitz auf dem rechten Wege. Das Wort Onpiov hatte mit dem Namen Trajan besonders für ein hebräisches Ohr eine gewisse phonetische Ähnlichkeit. Dabei spielte die Verschiedenheit des $\mathrm{T}$ keine Rolle. Aber, wie gesagt, mit dem hebräischen Alphabet läßt sich die Zahl nicht berechnen. Dagegen ergibt der Geschlechtsname Trajans, Ulpios, nach dem griechischen Alphabet die Zahl 666, wenn man das s am Schluß durch das Zahlzeichen für 6, das ihm sehr ähnlich ist, ersetzt. ${ }^{1}$ Der Name Ulpios ergiebt folgende Rechnung: $0=70, v=400$, $\lambda=30, \pi=80, \imath=10,0=70, \varsigma=6$. Der Name Ulpius ist der einzige Kaisername, der sich mit griechischen Buchstaben aus der Zahl 666 berechnen läßt. Gerade weil es nicht der bekanntere Name des Kaisers war, erhielt die Berechnung etwas Geheimnisvolles. Allerdings steht mir kein Material zur Verfügurig, um die Zulässigkeit der Verwendung des Zahlzeichens $\varsigma$ an Stelle des Schlußsigma zu beweisen. Ich mache aber darauf aufmerksam, daß auch bei der abgekürzten Schreibung der $Z$ ahl $\chi \xi_{\zeta}$ der erste und letzte Buchstabe, $\chi$ und $c$, als eine Abkürzung des Christusnamens aufgefaßt worden ist, was auch nur möglich ist, wenn man das Episemon, das Zahlzeichen für 6, mit dem Schlußsigma gleichsetzen würde. $\mathrm{Da}$ das Zahlzeichen für 6 kein Buchstabe ist, so mußte auf irgendeine Weise für gematrische Berechnungen ein Ersatz geschaffen werden, und was lag näher als die Nebenform des c, das Schlußsigma, das dem Episemon zum Verwechseln ähnlich ist, $z \mathrm{u}$

1 Nachträglich sehe ich, daß kein Geringerer als Grotius bereits Ulpios vorgeschlagen hat. 
verwenden? Damit wäre die isopsephisch-gematrische Gleichung gelöst: תריון $=666=0 u \lambda \pi$ เoc.

So allein erhält der 18 . Vers für die Leser der Offenbarung einen Sinn. Sie sollten wissen, wer das Tier ist, der Antichrist. Sie konnten es aus der Zahl des Tieres berechnen, denn es ist eines Menschen Zahl. Der Name ist in der Zahl des Tieres, 666, enthalten, es ist Ulpius Trajanus, - der Antichrist.

Dennoch wird die Deutung der Zahl 666 auf Nero nicht ohne weiteres abzulehnen sein. $\mathrm{Da} B$ der Verfasser der Apokalypse ein jüdischer Christ war, scheint mir unzweifelhaft zu sein. Für sich konnte er, um die Zahl żu finden, sich wohl des hebräischen Alphabetes bedienen. Auch die jüdischen Schriftgelehrten haben, wie Schlatter hervorhebt, aus den Namen die Zahlen berechnet, um herauszufinden, ob nicht Gott auf diese Weise ein Geheimnis offenbare (vgl. Schlatter, Offenb. S. 152; AT in der Ap S. 74). Hat er auf diese Weise aus dem Wort Onpiov durch das hebräische Alphabet die Zahl 666 gefunden, die sich ihm durch ihre besondere Form als ein Geheimnis aufdrängen mußte, so kann schon ihm durch das umgekehrte Verfahren der Name des Kaisers Nero offenbar geworden sein, zur Bestätigung seiner Überzeugung, daß Nero als der Antichrist wiederkehren werde. Diese hebräischen Deutungen und Berechnungen hatten aber in erster Linie nur für ihn einen Wert. Als sich dann aus dem griechischen Alphabet zugleich der Name Ulpios ergab, war die Beweiskette geschlossen und das gegeben, was für seine Leser wissenswert und wissensmöglich war. So ergibt sich die dreifache Gleichung:

Onpíov (in hebr. Buchstaben) $=666$

666 (in hebr. Buchstaben) = Kaiser Nero

666 (in griechischen Buchstaben) = Ulpios.

Da Johannes nach der bestimmten Nachricht des Irenäus bis in die Zeit Trajans lebte, ist ein Hinweis auf ihn, selbst von der Hand des Johannes, kein Ding der Unmöglịchkeit. Denkbar ist aber auch, wovon jetzt niçht weiter die Rede sein kann, daß v.17 von einem Herausgeber zur Zeit Trajans beigefügt worden ist.

Von diesem Gesichtspunkt wird auch die Variante 616 und die Deutung des Irenäus in gewissem Sinne bedeutsam. Gewiß ist die Zahl 616 nicht ursprünglich, sondern auf Grund einer Berechnung entstanden. Man hatte, wie oben angedeutet, die richtige Deutung verloren oder verworfen, weil sich Trajan doch nicht als der letzte Kaiser und auch nicht als der Antichrist erwies. Aber eines wußte man noch. Man wußte, daß die Zahl den Namen eines römischen Kaisers bedeuten 
mußte. So rechnete man in der richtigen Weise mit griechischen Buchstaben nach und fand in 666 das Wort $\Lambda \alpha \tau \epsilon i v o c$, das man als einen lateinischen Kaiser deutete. Man fand aber auch in dem Bilde des tötlich Verletzten die Züge des Caesar Gaius und setzte den Zahlenwert seines Namens 616 als die bessere Zahl in den Text ein. Eine andere Möglichkeit wäre, daß die Zahl 616 für Caligula selbständig entstanden wäre. Es wäre das ein Beweis, daß die Berechṇung der Kaisernamen in Zahlen bei den Juden üblich war. (Schlatter, a. a. O. S. 75.) Beide Deutungen beweisen, was man suchen wollte und wie man rechnen sollte.

Daß geschichtlich und sachlich die Deutung der Zahl 666 auf Trajan sich rechtfertigen läßt, kann nicht bestritten werden.

Im Jahre 96 wurde Domitian ermordet, und zwar infolge einer Verschwörung seiner nächsten Umgebung, worauf der Senat seine zahlreichen Ehrendenkmäler zerstörte und auf den Inschriften seinen Namen tilgte. Die Prätorianer, empört über die Ermordung Domitians, meuterten und nahmen Rache an der Mördern Domitians. Zu ihrer Versöhnung adoptierte Nerva den beliebten M. Ulpius Trajanus 97 n. Chr. Dio Cassius erwähnt, daß dem Trajan schon im Jahre 91 bei der Über-

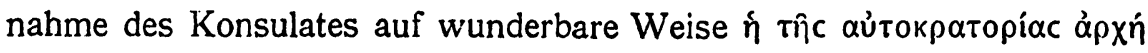
geweissagt worden sei. Sein Vater, M. Ulpius Trajanus, nahm als Legat der 10. Legion am jüdischen Kriege teil, und eroberte Japha. Die Reste der Verteidiger wurden auf grausame Weise bestraft. Er war an der Einnahme von Tiberias beteiligt, kämpfte bei Taricheä und unterwarf Peräa. Er galt als harter Kriegsmann, der dem Feinde gegenüber schonungslos verfuhr. Im Jahr 76 war er Legat in Syrien, von wo aus er mit seinem Sohne die Parther bekämpfte; 79 Prokonsul in Asien. Er starb vor dem Jahre 100 und wurde 112-114 ebenfalls theosiert. Münzen zeigen die Umschrift DIVVS PATER TRAIAN. Die Familie, insonderheit der Vater Trajans, war somit im Osten, besonders in Syrien und Kleinasien, zur Genüge bekannt, so daß man von dem Sohne dieses Bekämpfers der Juden nichts Gutes erwarten konnte. Ich verweise auf Dierauer, Beiträge zu einer krit. Geschichte Trajans (Leipz. 1868).

Das Reich war durch die Ermordung Domitians buchstäblich erschüttert, da nach Dierauer Domitian der Liebling der Soldaten war, und nach seinem Tode Empörungsversuche nur mit Mühe zurückgehalten werden konnten. Ein Jahr nach Nervas Amtsantritt brach der Sturm aus. Vor Nervas Augen wurden die Mörder Domitians niedergemacht. Die Verkündigung der Adoption Trajans war die Rettung. 
Confugit in sinum tuum (d. h. Trajans) concussa res publica (Plinius). Auf diese Zeit kam vom Heer in Pannonien ein Lorbeerkranz für den kapitolinischen Jupiter nach Rom als Zeichen eines Sieges. „So hatten es die Götter gefügt, daß das Symbol des Sieges die Erhebung eines unüberwindlichen Kaisers schmückte" Plin. Pan. c. 8. Man ist wirklich beinahe versucht, den Satz von dem tötlich verwundeten Haupt und seiner Heilung auf die Ermordung Domitians und die Erhebung Trajans zum Kaiser $z u$ beziehen. Jedenfalls kann man es verstehen, wenn Christen auf den Gedanken gekommen sind, in ihm den wiederkehrenden Nero zu erblicken, der vollenden würde, was Nero begonnen hatte.

Es ist nun $\mathrm{zu}$ untersuchen, ob in der Offenbarung sonst noch eine Stelle auf Trajan weist oder bis in die Zeit Trajans führt, weil dieser Punkt für die Entscheidung der Frage, ob wir in Vers 18 eine Glosse vor uns haben, von großer Wichtigkeit ist.

Die Reihen der gekrönten Hörner und der Häupter können, je nachdem man mit der Zählung einsetzt, bis auf Trajan herabgeführt werden. Trajan ist ohne Zählung der Kaiser des. Interregnums der 11. Kaiser von Cäsar an, der 10. von Augustus an. Doch liegt darin kein stringenter Beweis. Wesentlicher ist: Trajan ist das achte Haupt, von Nero an gerechnet, wenn man den im Orient nicht anerkannten Galba wegläßt: Nero, Otho, Vitellius, Vespasian, Titus, Domitian, Nerva, Trajan. Nerva regierte nur kurze Zeit. Ist c. 17 unter Domitian geschrieben wie das übrige Buch, so ist die Erwartung, daß nach der kurzen Regierungszeit eines letzten, siebenten Herrschers der Antichrist kommen müsse, auf Grund der unter Domitian einsetzenden neuen Feindseligkeiten des römischen Staates gegen die Christen psychologisch weit verständlicher als unter Vespasian. ${ }^{1}$ In die Zeit von 93-95 fällt auch die von Dio Cassius erwähnte Weissagung über Trajan. Doch ist dieser Vorfall schlecht bezeugt. Erst seine Erhebung unter Nerva

1 Für die Zählung der Reihe der 7 Häupter von Nero an kann folgendes geltend gemacht werden: a) Sie entspricht der allgemeinen Annahme, daB die Apc unter Domitian geschrieben ist. $\mathrm{Er}$ ist dann der 6 . Kaiser, so daB es nicht nötig ist, dieses Kapitel früher anzusetzen. b) In 13, 3 kann die Kardinalzahl $\mu$ iav als Ordinalzahl verstanden werden (Gunkel und Clemen). Man vgl. Ap 6,1 und 9,12. c) Der Beginn der Zählung von Nero an rechtfertigt sich dadurch, daß erst mit ihm der antichristliche Zug in die Politik des Reiches hineinkommt. Er ist der erste und dann wieder der achte, vgl. Spitta Off. S. 187. d) Wie Düsterdieck mit Recht hervorhob, hat man nicht von einem ersten an vorwärts gerechnet, z. B. von Caesar oder Augustus an, sondern rückwärts von dem gegenwärtigen Zeitpunkt an, d. h. von der Regierung des 6 . Königs. Denn man mußte mit der gegebenen Zahl von 7 Häuptern auskommen.

Unentschieden muß die Frage auf alle Fälle bleiben, ob in $17,10.11$ ein vaticinium ex eventu vorliegt, oder ob man schon unter Domitian den kommenden starken 
machte ihn bekannt. Bei einem Besuch Trajans auf dem Kapitol vor seiner Abreise in die Provinz ertönte aus der Volksmenge der Ruf: gegrüßet seist du, Imperator! Schon nach der bisherigen Annahme der Entstehung der Apokalypse dürfte dieselbe nicht weit von der Zeit entstanden sein, in welcher der Aufstieg Trajans begann. Weitere positive Zeugnisse besitzen wir allerdings nicht. Voelter glaubt zwar, daß die in dem Sendschreiben erwähnten Christenverfolgungen, insonderheit des Martyrium des Antipas, in die Zeit der trajanischen Christenverfolgung fallen (Entstehung 'der Ap. S. 35), freilich ohne einen Beweis für diese Behauptung bringen zu können. Sie ist aber nicht unmöglich. Das ist ja keine Frage, daß Christenverfolgungen der Abfassung des Buches unmittelbar vorangegangen sind, und daß sie mit dazu beigetragen haben, unter den Christen die Gewißheit der Nähe der Endzeit hervorzurufen und $\mathrm{zu}$ befestigen. Leider wissen wir $\mathrm{zu}$ wenig von den letzten Dezennien des ersten Jahrhunderts, um die Christenverfolgungen, welche die Apokalypse voraussetzt, chronologisch fixieren zu können.

Interessant ist die Ansicht Bengels (Erklärte Offenbrg. Joh. S. 253), der neben allerlei phantastischen Erklärungen der Offenbarung gelegentlich auch ein klares und nüchternes Verständnis für die zeitgeschichtlichen Beziehungen des Buches bewies. Bengel, der die Entstehung der Offenbarung in die Zeit nach Domitian, etwa ins Jahr 96 unter Nerva, verlegt, erklärt: „Dieses Kaisers Trajans Regierung ist von so sonderbar großer Wichtigkeit, daß man sie bei der Erfüllung dessen, was bald geschehen sollte, nicht überhüpfen kann, sondern vielmehr eben seinen Antritt zu Rom billig für den Anbruch der sieben Siegel halten soll." So bezieht Bengel nun die Worte der Siegelvision: diesem Reiter ward die Krone gegeben, auf Trajans Erhebung zum Mitregenten Nervas. „Trajanus hätte seiner Herkunft nach keine Hoffnung zu solcher Würde haben können, aber wegen seiner fürstlichen Qualitäten ward er von Nerva adoptiert; und da gewann es mit dem Kaisertum und dem ganzen Reich ein anderes Ansehen als vorhin, allermeist gegen den Orient. Ehe er selbst dahin auszog, brachte Palma, Landvogt in Syrien, das steinigte Arabien unter die römische Gewalt, und schon damals fanden sich die Gesandtschaften vieler Nationen, auch aus Indien, bei Trajano ein." So macht Bengel auch die vielen siegreichen Feldzüge Trajans im Orient für seine Deutung geltend. Zwischen Vespasian und Trajan habe man mehr vom Frieden sprechen hören als vom Krieg.

Mann ahnen konnte. Am leichtesten würde sich die Schwierigkcit lösen, wenn man diese Verse in die Zeit Nervas ansetzen dürfte. DaB man von dem alten Kaiser schon bei seiner Erhebung wissen konnte, $\mathrm{da} B$ er nicht lange bleiben werde, ist klar. 
Als aber Trajan zur Regierung kam, "sei der Friede von der Erde weggenommen gewesen". Ferner habe es unter Trajan eine schwere Hungersnot gegeben, besonders in Ägypten, so daß Trajan nach dem Zeugnis des Plinius den Ägyptern mit Getreide habe aushelfen müssen (100 n. Chr.). Der römische Historiker Sextus Aurelius Victor schreibt: eo tempore multe perniciosius quam sub Nerva Tiberis inundavit, magna clade aedium, et terrae motus gravis per provincias multas, atroxque pestilentia famesque et incendia facta sunt, quibus omnibus Trajanus per exquisita remedia plurimum opitulatus est... unde merito pater patriae dictus est. Man könnte für die Beziehung von Ap 6,2 auf Trajan daran erinnern, daß er den Beinamen Parthicus erhalten hat. Der Bogen war das Symbol. Doch führt das in eine späte Zeit. Immerhin sind alle diese Erwägungen Bengels im allgemeinen richtig und auf Tatsachen beruhend. Die glänzende Schilderung der Stadt Rom und des Aufschwungs von Handel und Schiffahrt in c. 17 und 18 entspricht durchaus dem Rom zur Zeit Trajans, im Gegensatz zu dem Niedergang Roms vor dem Ende Domitians, so daß Trajan für die Römer zum wahren Abgott wurde: ob haec per orbem terrarum Deo proximus nihil non venerationis meruit et vivus et mortuus.

\title{
Golgatha und das Prätorium des Pilatus.
}

\author{
Von Lic. theol. Carl Sachsse in Göttingen.
}

Bei Untersuchung der Frage nach der Lage der Leidensstätten Jesu in Jerusalem kann es sich nicht darum handeln, dieselben historisch einwandfrei $z u$ fixieren, da uns fast alles fehlt, was einen auch nur einigermaßen sicheren Schluß darüber erlauben könnte: die biblischen Nachrichten sind außerordentlich spärlich, aus der gleichzeitigen Profanliteratur ist garnichts $\mathrm{zu}$ entnehmen, und die Ausgrabungen haben, dank der gründlichen Zerstörung der Stadt im Jahre 70, nur wenig Licht in das Dunkel- werfen können. Unsere Aufgabe muß sich also darauf beschränken, zu prüfen, ob überhaupt die Möglichkeit vorliegt, daß die Leidensstätten des Herrn die von der Tradition als solche bezeichneten Örtlichkeiten gewesen sind.

\section{Golgatha.}

Wie bekannt, berichten die Evangelien, daß die Kreuzigung an einem Ort außerhalb der Stadt, ${ }^{1}$ aber nahe bei derselben ${ }^{2}$ vollzogen

\footnotetext{
1 EEárouar Mc 15, 20 u. Par.; vgl. Hebr 13,12. 2 Joh 19, 20.
} 